\title{
Functional consequences of a rare missense BARD1 c.403G >A germline mutation identified in a triple-negative breast cancer patient
}

\author{
Yuanting Zheng ${ }^{1+}$, Bingying $\mathrm{Li}^{1+}$, Dejing Pan ${ }^{2+}$, Jun $\mathrm{CaO}^{3}$, Jian Zhang ${ }^{3}$, Xiaolin Wang ${ }^{1}$, Xiangnan $\mathrm{Li}^{1}$, Wanwan Hou', \\ Ding Bao', Luyao Ren', Jingcheng Yang ${ }^{1}$, Shangzi Wang', Yangyang Qiu', Fei Zhou², Zhiwei Liu², Sibo Zhu', \\ Lei Zhang ${ }^{1}$, Tao Qing ${ }^{1}$, Yi Wang ${ }^{1}$, Ying $\mathrm{Yu}^{1 *}$, Jiaxue $\mathrm{Wu}^{4^{*}}$, Xichun $\mathrm{Hu}^{3^{*}}$ and Leming Shi ${ }^{1,5,6^{*}}$ (D)
}

\begin{abstract}
We identified a rare missense germline mutation in BARD1 (c.403G>A or p.Asp135Asn) as pathogenic using integrated genomics and transcriptomics profiling of germline and tumor samples from an early-onset triplenegative breast cancer patient who later was administrated with a PARP inhibitor for 2 months. We demonstrated in cell and mouse models that, compared to the wild-type, (1) c.403G>A mutant cell lines were more sensitive to irradiation, a DNA damage agent, and a PARP inhibitor; (2) c.403G>A mutation inhibited interaction between BARD1 and RAD51 (but not BRCA1); and (3) C.403G>A mutant mice were hypersensitive to ionizing radiation. Our study shed lights on the clinical interpretation of rare germline mutations of BARD1.
\end{abstract}

Keywords: BARD1, Rare mutation, c.403G>A, p.Asp135Asn, Integrated genomics profiling, Triple-negative breast cancer, Irradiation, DNA damage, PARP inhibitor, Functional assay

Incorporation of next-generation sequencing into clinical practice continues to expand the list of variants of unknown significance (VUS), making it challenging to appropriately interpret the clinical significance of such rare mutations in terms of pathogenicity and treatment options for the patient [1-3]. Analysis of the wholegenome sequencing data from a triple-negative breast

\footnotetext{
* Correspondence: ying_yu@fudan.edu.cn; jiaxue@fudan.edu.cn; huxichun2017@163.com; lemingshi@fudan.edu.cn

†Yuanting Zheng, Bingying Li and Dejing Pan contributed equally to this work.

${ }^{1}$ State Key Laboratory of Genetic Engineering, School of Life Sciences and Shanghai Cancer Center, Fudan University, Shanghai, China

${ }^{4}$ State Key Laboratory of Genetic Engineering, School of Life Sciences and Zhongshan Hospital, Fudan University, Shanghai, China

${ }^{3}$ Department of Medical Oncology, Fudan University Shanghai Cancer Center, Shanghai, China

Full list of author information is available at the end of the article
}

cancer (TNBC) patient's germline DNA uncovered a c.403G >A (p.Asp135Asn) mutation in BARD1 (Additional file 1). The BRCA1-associated ring domain 1 (BARD1) protein is a binding partner of BRCA1 and is essential for DNA damage repair [4], which is reported to be associated with breast cancer susceptibility $[2,3]$. In contrast to BRCA1 and BRCA2, for which numerous pathogenic mutations and benign variants have been identified [5], validated, and used for clinical decisionmaking, the clinical significance of a specific BARD1 mutation remains unclear and is classified as VUS [6]. The newly identified locus is located near the ring domain of BARD1 and highly conserved across different species (Fig. 1a). The germline variant was validated by orthogonal Sanger sequencing (Fig. 1b), and it was inherited from the father of the patient (Fig. 1c). This c.403G $>$ A mutation was only reported in one case from 
A Conserved p. D135N mutation

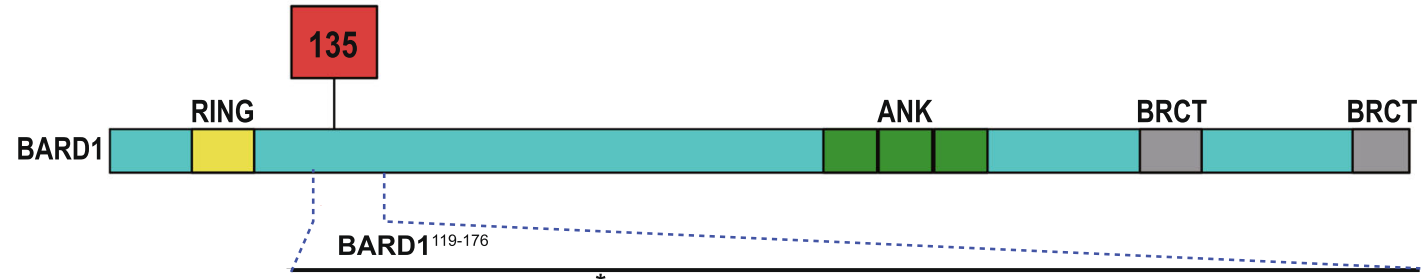

Homo_sapiens

Equus caballus

119 ELSDLKEDKPRKSLFND苂AGNKKNS I KMWF SPRSKKVRYVVSKASVQTQPAIKKDAS - AQ 176

Canis_caballus

66 ELSDSKEDTPGKG LFNDAENKKNS I KMWF SPR SKKVRYVVTKVSVQTQPQM I NDENSQQ 124

69 KL SDLKEDT SRENLFNDAENKKN S KMWF SPR SKKVRYVVSKVSVQTHPQV I SDENARQ 127

Bos_taurus

114 DLSDLKEKT SRKSLFNDAQSKKN I KMWF SPR SKKVRYTVSKLSVQTQPSVKNDENAQQ 172

Mus_musculus

113 K - - DSKDNT SRAS LFGDAERKKN S I KMWF SPRSKKVRYVVTKVSVQTQPQKAKDDKAQE 169

$$
\text { :Del4AA-: }
$$

B BARD1 Sequence

C Pedigree

Unaffected family member

Patientc. 403G>A, p. Asp135Asn
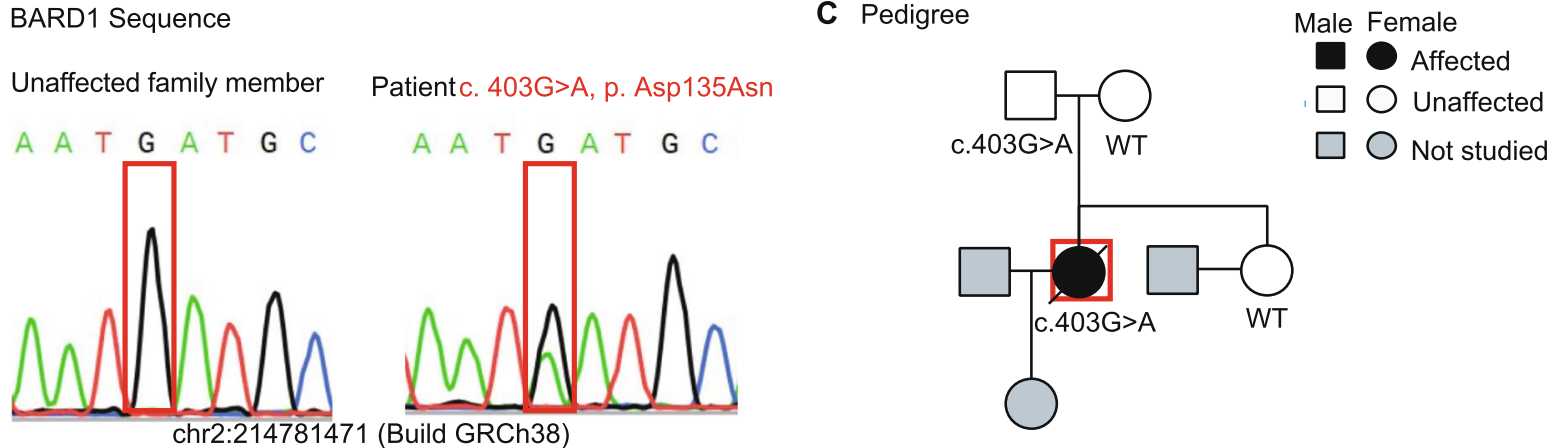

D Genomic profiling of tumor

E mRNA subtyping
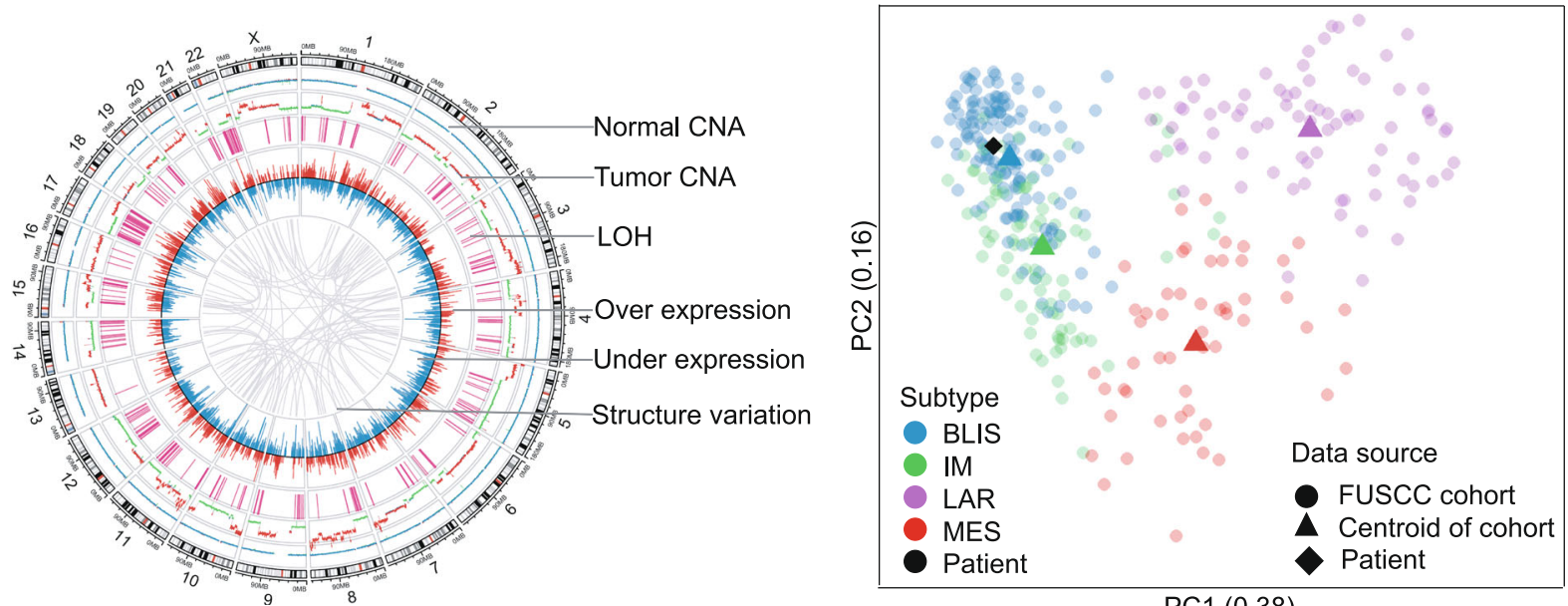

PC1 (0.38)

F BARD1 mutation profiles

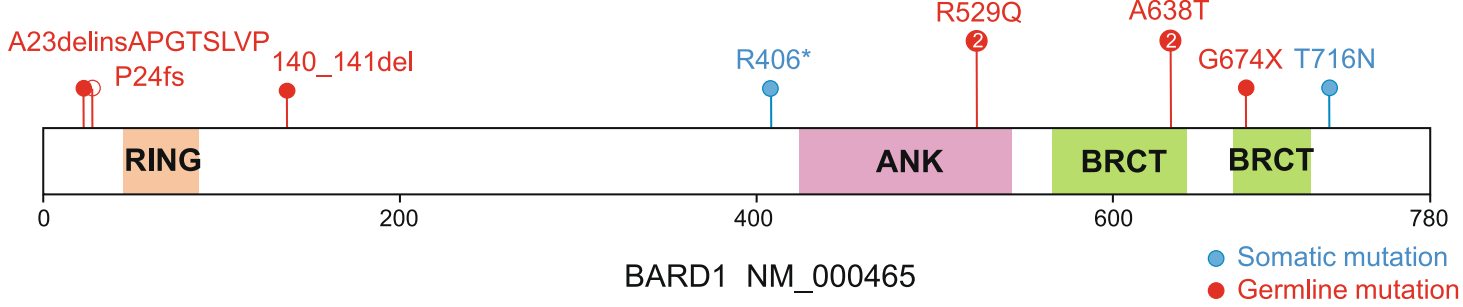

Fig. 1 (See legend on next page.) 
(See figure on previous page.)

Fig. 1 Genomic and transcriptomic profiling reveals a highly conserved, rare, and potentially pathogenic germline mutation (c.403G>A) of BARD1 in an early-onset TNBC patient that displayed a homologous recombination deficiency (HRD) somatic mutational signature. a A multiple sequence alignment of five mammalian BARD1 proteins. The highlighted residue 135, Asp (D), is conserved across all the species. $\mathbf{b}$ Chromatograms of the DNA sequences of the mutated locus by Sanger sequencing. $\mathbf{c}$ Genetic testing results of the patient family. $\mathbf{d}$ Genomic profiles of tumor from the patient. Large-scale copy number variations, loss of heterozygosity, and large-scale structural variations indicated an HRD somatic mutational signature. e The mRNA profile of the patient clustered together with the basal-like and immune-suppressed (BLIS) subgroup in a large TNBC cohort of FUSCC. $\mathbf{f}$ Somatic and germline mutation profiles of BARD1 in the FUSCC cohort. Germline variants are colored in red, and somatic mutations are colored in blue. Mutations marked with 2 indicates the mutation was detected twice in the TNBC cohort

138,632 whole-exome and whole-genome sequences in the Genome Aggregation Database (gnomAD [7]) with no reports on its clinical relevance. We predicted the pathogenicity of the rare missense c.403G $>$ A mutation using four computational tools with conflicting prediction results: tolerated by SIFT [8] and benign by PolyPhen 2 [9], while damaged by Mutation Assessor [10] and SNPs\&GO [11].

Importantly, computational analysis of the genomics profiling from the patient's tumor samples showed that the patient's tumor exhibited extensive copy number changes, loss of heterozygosity, and large-scale structural variations across the entire genome (Fig. 1d), indicating a typical genomic mutational signature resulting from deficiency in homologous recombination DNA damage repair [12]. Moreover, the mRNA expression profile of the tumor clustered closely with the basal-like and immune-suppressed (BLIS) subgroup in a large cohort of 465 TNBCs from Fudan University Shanghai Cancer Center (FUSCC) (Fig. 1e) [13], a study partially inspired by the patient. In addition, the mutation profile of BARD1 in the FUSCC TNBC cohort is shown in Fig. 1f, indicating that the germline mutation rate in BARD1 is low and the mutation loci were heterogeneous. Taken together, we hypothesized that the c.403G $>$ A mutation in BARD1 might be damaging by impairing the homologous recombination capacity of the cells.

To explore the in vitro functions of the c.403G $>A$ (p.Asp135Asn) mutation in BARD1, reconstitution with wild-type BARD1 (BARD1 ${ }^{\mathrm{WT}}$ res) and mutant BARD1 $\left(B A R D 1^{\mathrm{D} 135 \mathrm{~N}}\right.$ res) was conducted in two BARD1 knockout breast cancer cells (T47D and MDA-MA-468) by the CRISPR-Cas9 system. As shown in Fig. 2a, the BARD1 protein was undetectable in the knockout cells, whereas comparable expression of the BARD1 protein was detected in rescued cells $\left(\mathrm{BARD} 1^{\mathrm{WT}}\right.$ res and BARD1 ${ }^{\mathrm{D} 135 \mathrm{~N}}$ res) by Western blot using anti-BARD1 antibodies.

Impairment of DNA damage repair was reflected by increased sensitivity of tumor cells to DNA damage. In the $\gamma$-irradiation (IR) induced DNA damage experiment, BARD1 knockout T47D and MDA-MB-468 cells showed significantly reduced clonogenic survival compared with control cells after irradiation (Fig. $2 b, P<0.01$ ). As expected, ectopic expression of BARD $1{ }^{\mathrm{WT}}$ res resulted in full rescue of the reduced clonogenic survival, whereas ectopic expression of BARD $1^{\mathrm{D} 135 \mathrm{~N}}$ res showed partial rescue and the clonogenic survival was still significantly reduced compared with control cells (Fig. 2b). Similar results were observed in the DNA damage experiment with DNA inter-strand crosslinking agent mitomycin $\mathrm{C}$ (MMC). BARD1-deficient cells expressing BARD $1^{\text {D135N }}$ res were more sensitive to MMC than cells expressing wild-type BARD $1{ }^{\mathrm{WT}}$ res as determined by the CCK8 cell proliferation assay (Fig. 2b). Moreover, BARD1-deficient cells expressing BARD1 ${ }^{\mathrm{D} 135 \mathrm{~N}}$ res were also more sensitive to an investigational PARP inhibitor HS-10160 than cells expressing BARD1 ${ }^{\mathrm{WT}}$ res by the CCK8 cell proliferation assays (Fig. 2b).

Mechanically, BARD1 was thought to play an important role in DNA damage repair through direct interaction with BRCA1 by forming a BARD1-BRCA1 heterodimer [14]. However, immunoprecipitation (IP) assays showed that the c.403G $>$ A mutation in BARD1 had little effect on the interaction between BARD1 and BRCA1 (Fig. 3a). Recently, Zhao et al. reported that the BARD1 region (123-261) is indispensable for the interaction between BARD1 and RAD51, and RAD51mediated homologous DNA pairing [15]. This raises the possibility that the p.D135N germline mutation may affect the function of BRAD1 in DNA damage repair with RAD51-mediated homologous recombination pairing. On the other hand, we found that the interaction between BARD $1^{\text {D135N }}$ res and RAD51 was significantly weaker compared to that between BARD $1^{\mathrm{WT}}$ res and RAD51 in T47D and HEK293T cells after irradiation (Fig. 3b). Taken together, these data suggested that the c.403G $>$ A mutation in BARD1 increased sensitivity to PARP inhibitor treatment in vitro, which may be caused by the reduction of its DNA damage-induced association with RAD51.

To investigate the functions of the c.403G $>$ A mutation in BARD1 in vivo, two individual mouse lines (PM\#5 and $\mathrm{PM} \# 8$ ) with a point mutation c.718G $>\mathrm{A}$ in mice (p.Asp127Asn) corresponding to the c.G403A (p.Asp135Asn) mutation in human BARD1 were obtained using the CRISPR/Cas9 system. Mice with the c.718G>A point mutation (PM\#5 and $\mathrm{PM} \# 8$ ) were significantly more 
A

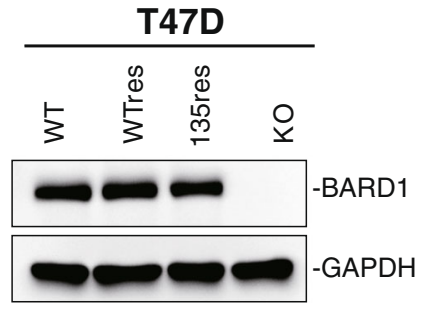

B

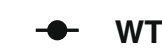

WT

T47D
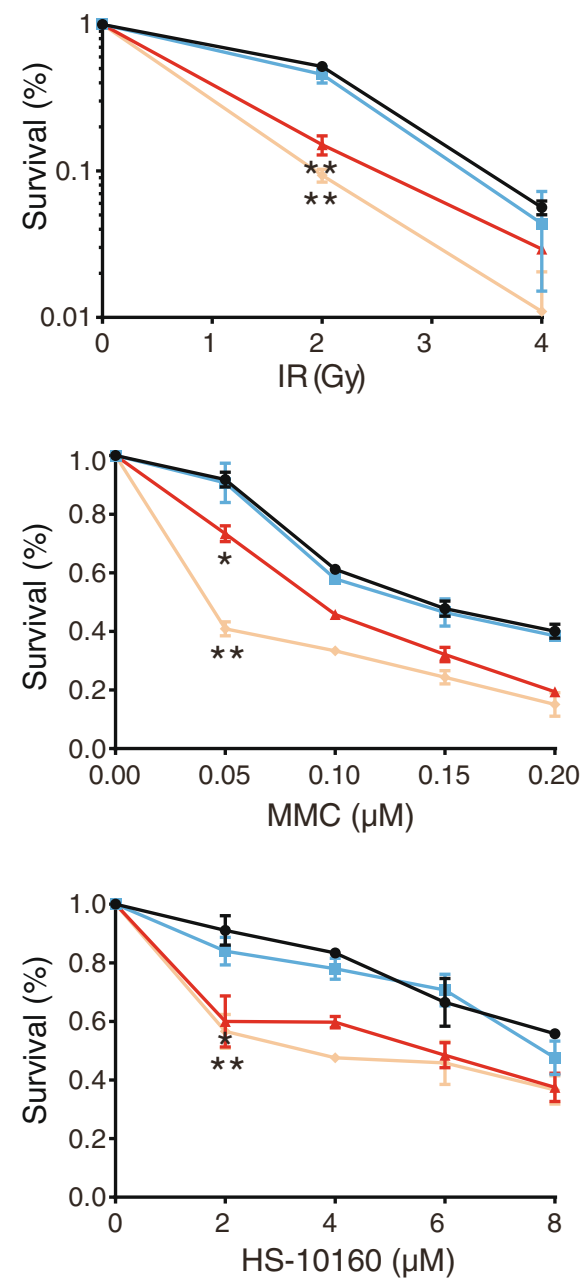

MDA-MB-468

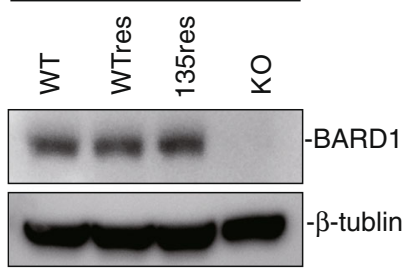

$\dashv$ WTres $\quad \leftarrow$ 135res
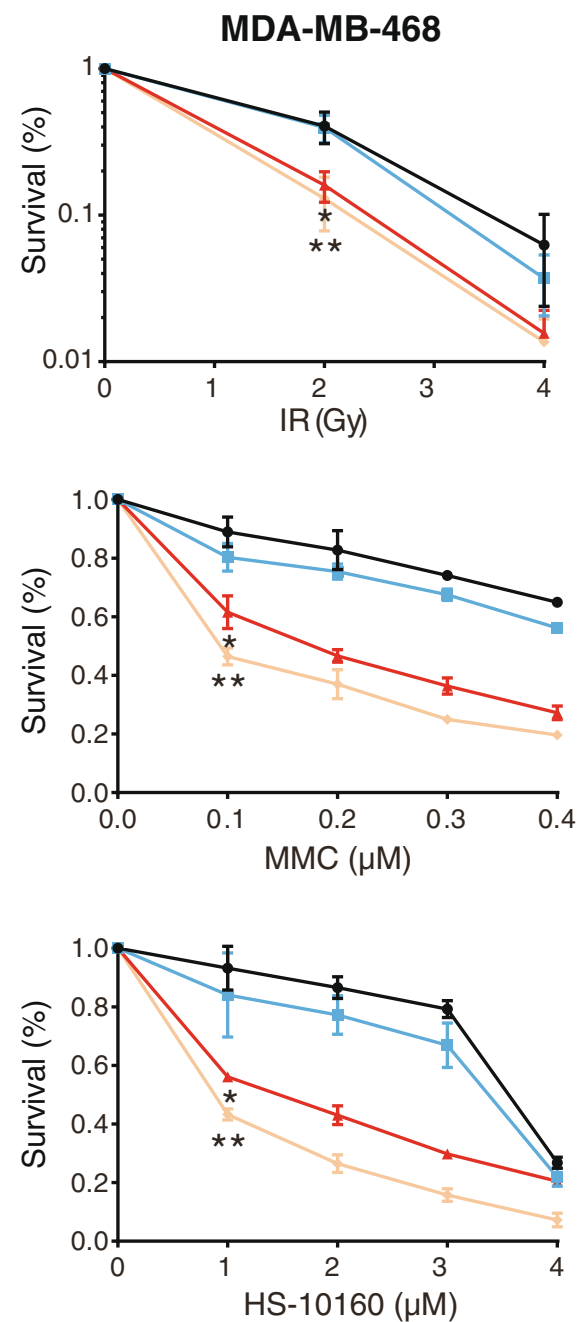

Fig. 2 BARD1 p.Asp135Asn mutation increased cell sensitivity to DNA damage in vitro. a Western blot testing of the Flag-tagged BARD1 expression in BARD1 knockout (KO), wild-type BARD1 ${ }^{\text {WT }}$ rescue (WTres), and BARD1 ${ }^{135}$ rescue (D135Nres) breast cancer cell lines. b Clonogenic survival of T47D cells (left) and MDA-MB-468 (right) cells expressing BARD1 ${ }^{W T}$ res or BARD1 ${ }^{135}$ res after $\gamma$-irradiation (IR, up), and cell survival (CCK8 assay) of T47D cells (left) and MDA-MB-468 cells (right) expressing BARD $1^{\text {WT }}$ res or BARD ${ }^{135}$ res after treatment with mitomycin $C$ (MMC, middle) and PARP inhibitor (HS-10160, down). Data are means \pm s.d, $n=3$. $P$ values were calculated using a two-tailed Student's $t$-test. ${ }^{*} P<0.05$, ${ }^{* *} P<0.01$

susceptible to irradiation than the wild-type mice (Fig. 4a, b). With a sub-lethal dose (7 Gy) (i.e., 10\% of the LD50 for wild-type C57BL6J male mice) of total body irradiation (TBI), the time to reach $50 \%$ lethality for wild-type control mice was nearly 4 days or longer compared to the homozygotes point mutation lines, and the $P$ values of log-rank tests all showed a statistically significant difference $(P=$ 0.045 for $\mathrm{PM} \# 5$ and 0.025 for $\mathrm{PM} \# 8$ ). 

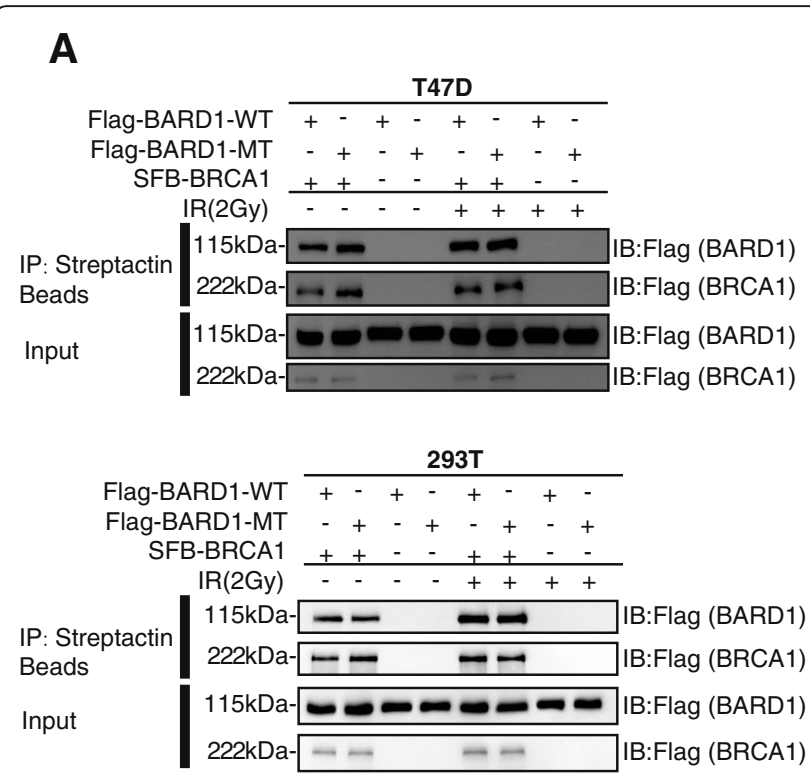

B
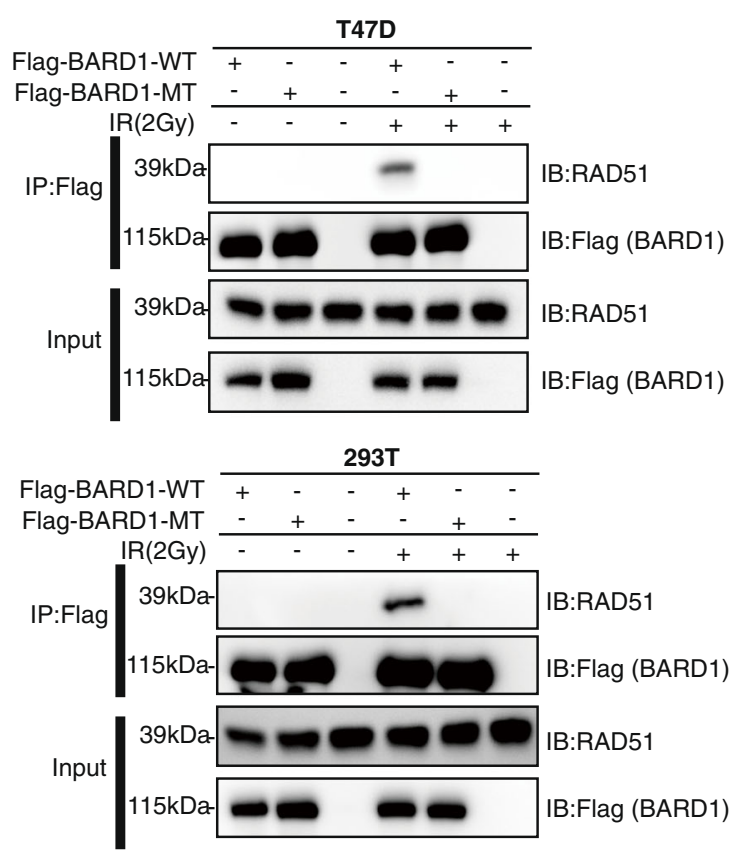

Fig. 3 BARD1 p.Asp135Asn mutation impaired the DNA damage-induced association with RAD51 in vitro. a Western blot (WB) verification of the effect of BARD1MT (p.Asp135Asn) on BARD1-BRCA1 binding before and after irradiation (2 Gy). FLAG-BARD1-WT/FLAG-BARD1-MT and SFB-BRCA1 were co-transfected into T47D and HEK293T cells and applied to immunoprecipitation followed by Western blot. Whole-cell lysates were also blotted and shown as input. $\mathbf{b}$ Western blot verification of the effect of BARD1-MT on BARD1-RAD51 binding in T47D and HEK293T cells. Cells expressing BARD1-WTres and BARD1-MTres were treated with irradiation (2 Gy) and then subjected to immunoprecipitation and Western blot

Furthermore, one line with a deletion of four amino acids (FGDA) indicated as \#121SRASL"FGDA"ERKKNSIKMW\#138 (referred to as Del4aa) and another line with a deletion of six amino acids (SLFGDA) indicated as \#121RK"SLFNDA"SRA"SLFGDA"ERKKNSIKMW\#138 (referred to as Del6aa) were also obtained and undergone with the same irradiation experiments. Similarly, the deletion lines of Del4aa and Del6aa displayed a statistically significantly lower survival compared to littermate wildtype control mice $(P=0.0006$ for Del4aa and 0.012 for Del4aa; Fig. 4c, d). This result provided strong evidence for a pivotal role of this short and conserved sequence to maintain BARD1 functions in mammals.

Taken together, both in vitro and in vivo biological validation data indicated potential clinical benefits of PARP inhibitors for patients carrying c.403G $>$ A mutation. However, no PARP inhibitor was marketed in China and BARD1 mutation was not used as a biomarker for enrollment of patients in clinical trials in China when the patient was diagnosed and treated. Eventually, the patient was administrated with an investigational PARP inhibitor for about 2 months as a compassionate use after oncologists exhausted all available treatment regimens. By clinical observations, the shrinkage of the primary breast tumor and the metastases to the bone and liver was obvious. No drug resistance was found during the short treatment period. Unfortunately, the patient was later on diagnosed with brain metastases, which might have already occurred before the initiation of PARP inhibitor treatment, and therefore treated with radiation therapy. However, serious anemia occurred during radiation therapy and the PARP inhibitor therapy was discontinued. It seems that incorporating radiation therapy into the treatment scheme was not advisable in such a situation due to increased sensitivity resulting from the rare germline mutation.

Our results added evidence that the inherited c.403G $>$ A mutation in the highly conserved functional domain of BARD1 appears to suggest a favorable response of a triple-negative breast cancer patient to a PARP inhibitor, thus benefiting patients beyond carriers of BRCA1 or BRCA2 germline mutations. Incorporation of next-generation sequencing into clinical practice continues to expand the list of VUS in DNA damage repair genes, posing particular challenges in the clinical decision for carriers of the VUS about personalized drug therapy and genetic counseling. Our integrated approach by combining the identification of germline mutation with somatic and transcriptomic profiling of cancer patients, 


\section{A Point Mutation-\#5 (Female)}

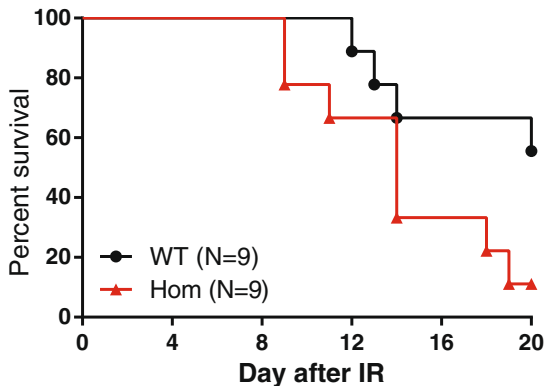

C 4 amino acids deletion

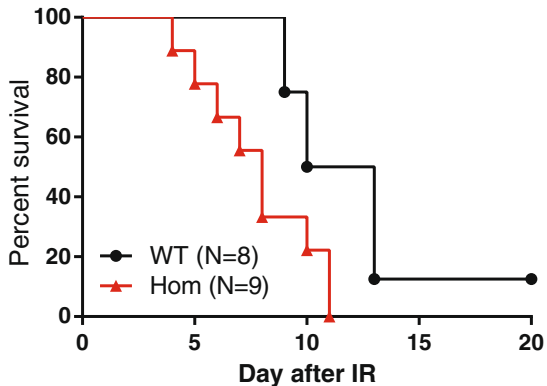

B Point Mutation-\#8 (Male)

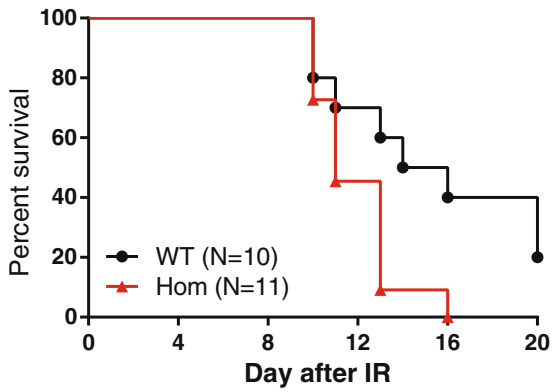

D 6 amino acids deletion

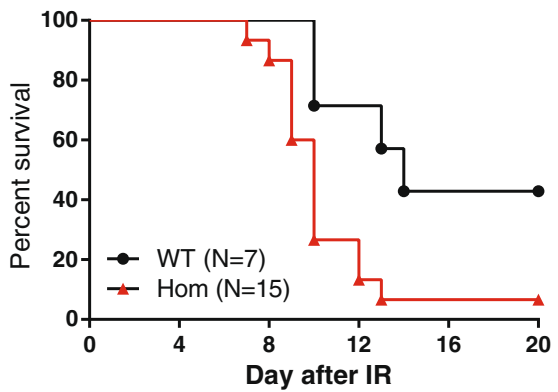

Fig. 4 BARD1 p.Asp127Asn increased sensitivity of mice to irradiation. a Point mutation D127N-\#5, b point mutation D127N-\#8, c four amino acid deletion, and $\mathbf{d}$ six amino acid deletion were exposed to a sub-lethal dose (7 Gy) of total body irradiation (TBI). The littermate wild-type mice were used as controls. Survival at day 20 after TBI was analyzed and shown as percent mortality on the $Y$-axis. Survival curves of wild-type (WT) and homozygous mutation (Hom) mice after sub-lethal irradiation were shown. $n \geq 7$ mice per genotype. $P$ values shown are for the Wald test

followed by functional assays at the molecular, cellular, and animal levels, will accelerate the interpretation of VUS for precision medicine.

\section{Abbreviations}

HR: Homologous recombination; IR: $\gamma$-irradiation; MMC: Mitomycin C; PARP: Poly ADP-ribose polymerase; VUS: Variants of unknown clinical significance; WT: Wild-type

\section{Supplementary Information}

The online version contains supplementary material available at https://doi. org/10.1186/s13058-021-01428-5.

Additional file 1. Study case, sample preparation, data generation, bioinformatics analyses, cell culture assays, development of mouse models with specific mutations, and in vivo irradiation assays with mutant mice were performed as described.

\section{Acknowledgements}

We thank Drs. David Huang, Xingxu Huang, and Lajos Pusztai and members of the laboratory for helpful discussions and/or critical reading of an earlier version of the manuscript, and the patient and family members for their cooperation.

\section{Authors' contributions}

L. S., X. H., J. W., and Y. Z. conceived and designed the overall project. Y. Z., B. L., X. W., W. H., D. B., Y. Q., S. Z., and L. Z. performed the in vitro experiments. D. P., F. Z., and Z. L. performed the in vivo experiments. Y. Y., X. L., L. R., J. Y., S. W.,T. Q., and Y. W. performed the omics data analysis. X. H., J. C. and J. Z perform the clinical data analysis. Y. Z., B. L., D. P., Y. Y., J. W., and L. S. wrote the manuscript. All authors reviewed and edited the manuscript. The authors read and approved the final manuscript.

\section{Authors' information}

Professor and Director, Center for Pharmacogenomics, School of Life Sciences and Shanghai Cancer Center, Fudan University, Shanghai, China.

\section{Funding}

Supported by grants from the National Natural Science Foundation of China (31720103909, 31671368, and 31771299), the National Key R\&D Project of China (2018YFE0201603, 2018YFA0801100, 2017YFC0907502, 2017YFC0907000, 2017YFF0204600, and 2016YFC0901704), Shanghai Municipal Science and Technology Major Project (2017SHZDZX01), Chinese Academy of Sciences' Major R\&D Project (XDA12040104), Shandong Province Key R\&D Project (2017CXGC1209), the Science and Technology Project of Jiangsu Province (BZ2020067), the National Center for International Research of Genomics (2017B01012), and the 111 Project (B13016).

Availability of data and materials

The datasets used and/or analyzed during the current study are available from the corresponding authors on reasonable request.

\section{Declarations}

Ethics approval and consent to participate

This study was approved by the independent ethics committee at Fudan University Shanghai Cancer Center. The patient and all participating family members provided written informed consent to participate in the study.

Consent for publication

Not applicable.

Competing interests

The authors declare that they have no competing interests. 


\section{Author details}

'State Key Laboratory of Genetic Engineering, School of Life Sciences and Shanghai Cancer Center, Fudan University, Shanghai, China.

${ }^{2}$ Cambridge-Suda Genomic Resource Center and Jiangsu Key Laboratory of Neuropsychiatric Diseases Research, Soochow University, Suzhou, China. ${ }^{3}$ Department of Medical Oncology, Fudan University Shanghai Cancer Center, Shanghai, China. ${ }^{4}$ State Key Laboratory of Genetic Engineering, School of Life Sciences and Zhongshan Hospital, Fudan University, Shanghai, China. ${ }^{5}$ Human Phenome Institute, Fudan University, Shanghai, China. ${ }^{6}$ Fudan-Gospel Joint Research Center for Precision Medicine, Fudan University, Shanghai, China.

Received: 18 September 2020 Accepted: 13 April 2021

Published online: 01 May 2021

\section{References}

1. Nielsen FC, van Overeem HT, Sorensen CS. Hereditary breast and ovarian cancer: new genes in confined pathways. Nat Rev Cancer. 2016;16(9):599_ 612. https://doi.org/10.1038/nrc.2016.72.

2. Couch FJ, Hart SN, Sharma P, Toland AE, Wang X, Miron P, et al. Inherited mutations in 17 breast cancer susceptibility genes among a large triple-negative breast cancer cohort unselected for family history of breast cancer. J Clin Oncol. 2015;33(4):304-11. https://doi.org/10.12 00/JCO.2014.57.1414.

3. D'Andrea AD. Susceptibility pathways in Fanconi's anemia and breast cancer. N Engl J Med. 2010;362(20):1909-19. https://doi.org/10.1056/NEJMra 0809889.

4. Irminger-Finger I, Jefford CE. Is there more to BARD1 than BRCA1? Nat Rev Cancer. 2006;6(5):382-91. https://doi.org/10.1038/nrc1878.

5. Cline MS, Liao RG, Parsons MT, Paten B, Alquaddoomi F, Antoniou A, et al. BRCA challenge: BRCA exchange as a global resource for variants in BRCA1 and BRCA2. Plos Genet. 2018;14(12):e1007752. https://doi.org/10.1371/journa l.pgen.1007752.

6. Li MM, Datto M, Duncavage EJ, Kulkarni S, Lindeman NI, Roy S, et al. Standards and guidelines for the interpretation and reporting of sequence variants in cancer: a joint consensus recommendation of the Association for Molecular Pathology, American Society of Clinical Oncology, and College of American Pathologists. J Mol Diagn. 2017;19(1):4-23. https://doi.org/10.101 6/j.jmoldx.2016.10.002

7. Lek M, Karczewski KJ, Minikel EV, Samocha KE, Banks E, Fennell T, et al. Analysis of protein-coding genetic variation in 60,706 humans. Nature. 2016; 536(7616):285-91. https://doi.org/10.1038/nature19057.

8. Ng PC, Henikoff S. SIFT: Predicting amino acid changes that affect protein function. Nucleic Acids Res. 2003;31(13):3812-4. https://doi.org/10.1093/nar/ gkg509.

9. Adzhubei I, Jordan DM, Sunyaev SR. Predicting functional effect of human missense mutations using PolyPhen-2. Curr Protoc Hum Genet. 2013; Chapter 7:Unit7 20.

10. Reva B, Antipin $Y$, Sander C. Predicting the functional impact of protein mutations: application to cancer genomics. Nucleic Acids Res. 2011;39(17): e118. https://doi.org/10.1093/nar/gkr407.

11. Calabrese R, Capriotti E, Fariselli P, Martelli PL, Casadio R. Functional annotations improve the predictive score of human disease-related mutations in proteins. Hum Mutat. 2009;30(8):1237-44. https://doi.org/10.1 002/humu.21047.

12. Davies H, Glodzik D, Morganella S, Yates LR, Staaf J, Zou X, et al. HRDetect is a predictor of BRCA1 and BRCA2 deficiency based on mutational signatures. Nat Med. 2017;23(4):517-25. https://doi.org/10.103 8/nm.4292.

13. Jiang YZ, Ma D, Suo C, Shi J, Xue M, Hu X, et al. Genomic and transcriptomic landscape of triple-negative breast cancers: subtypes and treatment strategies. Cancer Cell. 2019;35(3):428-40 e5. https://doi.org/10.1 016/j.ccell.2019.02.001.

14. Meza JE, Brzovic PS, King MC, Klevit RE. Mapping the functional domains of BRCA1. Interaction of the ring finger domains of BRCA1 and BARD1. J Biol Chem. 1999;274(9):5659-65. https://doi.org/10.1074/ jbc.274.9.5659.

15. Zhao W, Steinfeld JB, Liang F, Chen X, Maranon DG, Jian Ma C, et al. BRCA1BARD1 promotes RAD51-mediated homologous DNA pairing. Nature. 2017; 550(7676):360-5. https://doi.org/10.1038/nature24060.

\section{Publisher's Note}

Springer Nature remains neutral with regard to jurisdictional claims in published maps and institutional affiliations. 